\title{
2347. Numerical simulation and experimental study of aerodynamic noises for the cabin of the high-speed train
}

\author{
Ming Lv', Dong-er $\mathrm{Xu}^{2}$, Hua $\mathrm{He}^{3}$ \\ School of Mechanical and Vehicle Engineering, Bengbu University, Bengbu, 233000, China \\ ${ }^{1}$ Corresponding author

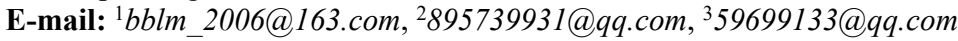

Received 6 May 2016; received in revised form 26 August 2016; accepted 29 August 2016

DOI https://doi.org/10.21595/jve.2016.17140

Check for updates

\begin{abstract}
For improving noises in the cabin quickly, large eddy simulation was adopted to compute the fluctuating pressure of the cabin surface numerically. Then, the fluctuating pressure was mapped to the boundary element model as the excitation loads to obtain the aerodynamic noise distribution of the cabin at different frequencies, which was then compared with the experimental result. There were some differences between the computational model and actual model, and the experimental results were more than the simulation result, which was within the acceptable range of the engineering. The comparative result indicated that the prediction model was reliable. In addition, as presented from the computational results: SPLs on cabin surface were changed between 60-110 dB, and greater SPLs were located on the transition position which was from the nose tip to the roof surface of the cabin. The aerodynamic noise on the cabin surface was mainly in low-frequency.
\end{abstract}

Keywords: high-speed trains, cabin, fluctuating pressure, aerodynamic noises.

\section{Introduction}

The dynamic environment of the high-speed train was changed into the aerodynamic force, which brought serious change and restriction of excessive noise pollution, therefore being rejected in a veto [1]. S2500 train reached $350 \mathrm{~km} / \mathrm{h}$ in both design and experimental speeds. However, it was limited by noises, and the train was only allowed to operate at the speed of $300 \mathrm{~km} / \mathrm{h}$. Therefore, the control of noises was the necessary requirement for the realization of the sustainable and development of the railway. Aerodynamic noises were increased with the sixth power of the train speed [2]. With the constant construction of the high-speed railway and further increase of the speed, the aerodynamic noise was becoming more and more prominent. Therefore, the research and reduction regarding aerodynamic noises has become the key to controlling the highspeed train noise. Through wind tunnel test and numerical simulation, the aerodynamic noise source and external fluctuating pressure of the high-speed train were studied and the analysis of the external sound field was conducted [3-5]. Liu solved the generalized Lighthill equation, obtained integral formulas of the aerodynamic noise, and made experimental research on the distribution and frequency characteristics of the fluctuating pressure in the wind tunnel [6, 7]. Liu also established mathematical and physical model for three-dimensional circumferential flow field regarding the head of the high-speed train, applied Lighthill acoustic analogy theory to compute the far-field aerodynamic noise of the high-speed train, and adopted a broadband noise source model to compute the aerodynamic noise source on the body surface of the high-speed train [8]. Xiao used the head surface of the high-speed train as the research object and analyzed aerodynamic noise characteristics of the head [9]. Yuan established a computational model including a head train and a tail train, in order to computing aerodynamic noise source intensity and far-field aerodynamic noises on the train surface [10]. A computational model including a head train, middle train and tail train was built in reference [11] to compute noise source intensity and far-field aerodynamic noise on the train surface, although the detailed analysis and summary did not consider bogies and far-field noises in the model. Sun established the aerodynamics model of the high-speed train, analyzed the flow field characteristics of the train head, compartment joint and train tail, and studied the contribution of different train parts on the aerodynamic noise, which 
ignored bogies and pantographs [12].

The mentioned study ignored the train floor and porous sound absorption materials when the aerodynamic noise model of the high-speed train was established, whose model accuracy was therefore not very high. Besides, the researched object was the entire train model, which would reduce the computational efficiency and increase the computational cost. Only aerodynamic noises of the high-speed train cabin were studied in the paper. When the aerodynamic noise model of the cabin was established, the interior trims of the train were considered, and then only the local model of the head was established for researches, which could improve the computational efficiency and cost.

\section{Computational method of aerodynamic noises of the high-speed train}

\subsection{Main transmission process of aerodynamic noises}

When the high-speed train was operating, the train interacted with the air. In several parts of the body, the airflow emerged separation vortex and vortex shedding, thus generating unsteady flow. Therefore, strong fluctuating pressure field was appeared on the external surface of the body. The fluctuating pressure acting on the exterior surface of the body was the reason of the interior aerodynamic noise [8]. Exterior fluctuating pressure and the induced aerodynamic noise had two main transmission ways to the train [9]: Firstly, exterior aerodynamic noises spread to the train through the door, windows and another gap directly. Secondly, external fluctuating pressure acted on the train panels, doors and windows, caused the vibration of panels, doors and windows and radiated noises to the interior space. When the closure condition of the train was good, the noise transmitted through holes and pores can be ignored. Therefore, the vibration was the main noise source. Two stages were conducted during the computation of the aerodynamic noise of the cabin: large eddy simulation was applied to compute the transient external flow field of the cabin surface and obtain the fluctuating pressure; and boundary element method was adopted to compute the aerodynamic noise.

\subsection{Control equations of large eddy simulation}

Large Eddy Simulation (LES) had the basic idea to compose the turbulent flow through different scales of eddies. And large-scale eddy played a major role in the generation of turbulence energy and Reynolds stress as well as diffusion of various turbulence amounts. The behavior of large eddy was strongly dependent on the boundary conditions, which varied in accordance with the flow type. However, small vortex had small impact on the trend of above functions, and the minimum eddy primarily played the dissipation role. At high Reynolds number, the small eddy was approximately isotropic and less affected by the boundary conditions, with greater commonality. Although dissipation scale cannot be computed through the current computer, inertial scale can be obtained. Therefore, N-S equations which were relevant to the discrete time could be applied to determine the behavior of big eddy, and more general model could be adopted to simulate the behavior of small vortex. Furthermore, the flow field speed variable $u_{i}$ in N-S equation was changed to the large scale through LES to solve the variable $\bar{u}_{i}$ directly. Thus, flow field variables can be decomposed into two parts of large scale and subgrid scale, as shown below:

$u_{i}=\bar{u}_{i}+u_{i}^{\prime}$

wherein: $u_{i}^{\prime}$ was the filtered subgrid scale, and $\bar{u}_{i}$ was the big eddy greater than the filter scale, which was obtained in the whole computational domain $D$ through the following convolution integral: 
$\bar{u}_{i}\left(x_{1}, x_{2}, x_{3}\right)=\iiint_{D}\left[\prod_{j=1}^{3} G_{j}\left(x_{j}, x_{j}^{\prime}\right)\right] u_{i}\left(x_{1}^{\prime}, x_{2}^{\prime}, x_{3}^{\prime}\right) d x_{1}^{\prime} d x_{2}^{\prime} d x_{3}^{\prime}$,

wherein: $x_{i}$ and $x_{1}^{\prime}$ were spatial coordinates, and $G$ was the filter function, which satisfied the following equation:

$\iiint_{D}\left[\prod_{j=1}^{3} G_{j}\left(x_{j}, x_{j}^{\prime}\right)\right] d x_{1}^{\prime} d x_{2}^{\prime} d x_{3}^{\prime}=1$.

The filter with volume-weighted function was most commonly applied in the finite difference method and finite volume method, namely:

$G_{j}\left(x_{j}, x_{j}^{\prime}\right)= \begin{cases}1 / \Delta_{j}, & \left|x_{j}-x_{j}^{\prime}\right| \leq \Delta_{j} / 2, \\ 0 & \left|x_{j}-x_{j}^{\prime}\right|>\Delta_{j} / 2,\end{cases}$

wherein: $\Delta_{j}(j=1,2,3)$ was the grid scale in three coordinate directions. After filtering, the obtained incompressible N-S equation form was as follows:

$\frac{\partial \bar{u}_{i}}{\partial t}+\frac{\partial\left(\overline{u_{i} u_{j}}\right)}{\partial x_{j}}=-\frac{1}{\rho} \frac{\partial \bar{p}}{\partial x_{i}}+v \frac{\partial^{2} \bar{u}_{i}}{\partial x_{k} \partial x_{k}}$

wherein: $\rho, v$ were air density and kinematic viscosity, respectively. $\bar{u}_{i}, \bar{p}$ were the filtered speed and pressure, respectively. As $\bar{u}_{i}$ and $\overline{u_{i} u_{j}}$ cannot be solved simultaneously, the following decomposition need to be made: $\overline{u_{i} u_{j}}=\bar{u}_{i} \cdot \bar{u}_{j}+\tau_{i j}$. Wherein, $\tau_{i j}$ was the subgrid shear stress tensor, $\tau_{i j}=\tau_{k k} \delta_{i j} / 3-2 \mu_{t} \bar{S}_{i j}$, and $\mu_{t}$ were subgrid turbulence viscosity. $\bar{S}_{i j}$ was the strain rate tensor determined by the solved large eddy, which can be expressed as follows:

$\bar{S}_{i j}=\frac{1}{2}\left(\frac{\partial \bar{u}_{i}}{\partial x_{j}}+\frac{\partial \bar{u}_{j}}{\partial x_{i}}\right)$

The incompressible N-S equation with continuity equation can be expressed as follows:

$$
\begin{aligned}
& \frac{\partial \bar{u}_{i}}{\partial x_{i}}=0, \\
& \frac{\partial \bar{u}_{i}}{\partial t}+\frac{\partial \bar{u}_{i} \bar{u}_{j}}{\partial x_{j}}=-\frac{1}{\rho} \frac{\partial \bar{p}}{\partial x_{i}}+v \frac{\partial^{2} \bar{u}_{i}}{\partial x_{k} \partial x_{k}}-\frac{1}{\rho} \frac{\partial \tau_{i j}}{\partial x_{j}} .
\end{aligned}
$$

In the application of Smagorinsky model, the subgrid turbulence viscosity can be expressed as the following equation:

$\mu_{t}=\rho L_{s}^{2}|\bar{S}|$,

wherein: $|\bar{S}|=\sqrt{2 S_{i j} S_{i j}}$, subgrid mixing length was $L_{s}=\min \left(k \delta, C_{s} V^{1 / 3}\right), k=0.42$ was a parameter of von Karman, $\delta$ was the distance to the nearest wall, $V$ was the volume of the computed element, $C_{s}$ was Smagorinsky constant, and $C_{s}=0.1$ can give a reasonable solution in a wide flow range. 


\subsection{Control equations of the acoustic boundary element method}

The displacement response of the train was a time-domain response under aerodynamic loads, which could obtain the frequency-domain response of the structural displacement after Fourier transformation. The sound field pressurepshould meet the Helmholtz equation:

$\nabla^{2} p-k^{2} p=-j \rho_{0} \omega q$

wherein: $k$ was the wave number, $j$ was the imaginary unit, $\rho_{0}$ was the air density, $\omega$ was the angular frequency, and $q$ was the volume velocity per unit volume.

In the fluid-solid surface $S$, the solution $p$ of Helmhotz equation should satisfy the following equation:

$\partial p / \partial n=-j \rho \omega v_{n}$

wherein: $n$ was the normal direction of the border, and $v_{n}$ was the normal speed on surface $S$.

The sound pressurepat any point of interior sound field of the train can be obtained through the integration of sound pressurepon surface $S$ and normal vibration velocity $v$, namely:

$p=C^{T} p+D^{T} v$

where: $C_{i}$ and $D_{i}$ were components of $C$ and $D$, respectively:

$C_{i}=\int_{S} N_{i} \times \frac{\partial G(X, Y)}{\partial n} d S$,

$D_{i}=j \rho_{0} \omega \int_{S} N_{i} \times G(X, Y) d S$,

wherein: $N_{i}$ was the shape function, which was 1 on node $i$ and 0 on other nodes. $G(X, Y)$ was the Green function, which should satisfy the Helmholtz equation.

\section{Computation of aerodynamic characteristics of the high-speed train}

The body structure of the high-speed train was consisted of the aluminum profile, as shown in Fig. 1. It included aluminum profiles of roof, floor and lateral side, respectively. Then, some sound absorption materials were installed on the aluminum profile to form the interior trims of the high-speed train, as shown in Fig. 2.

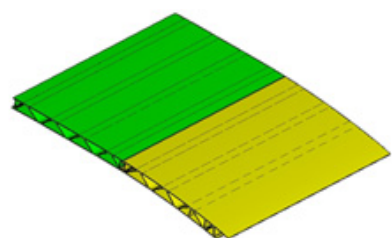

a) Roof aluminum profile

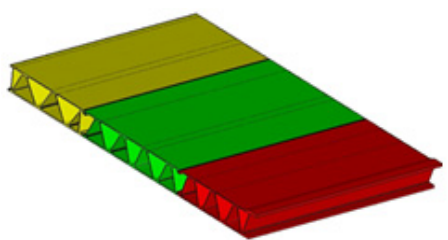

b) Floor aluminum profile

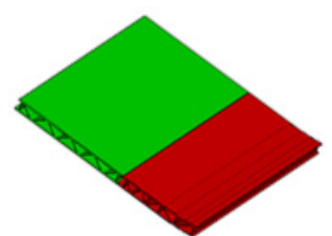

c) Lateral side aluminum profile

Fig. 1. Aluminum profiles of body structure

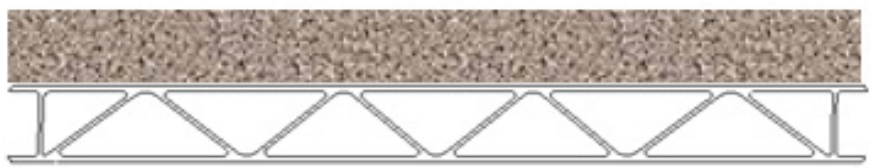

Fig. 2. Interior trims of the high-speed train 
The paper mainly researched aerodynamic noises on the surface of the high-speed train cabin and did not pay attention to noises in the passenger compartment. When the aerodynamic noise of the high-speed train was researched, appendixes such as bogies and pantographs were always ignored, so that the top and bottom parts of the train were approximated as plane structures. When flow passed through the train top and bottom, it can realize smooth transition and would not be influenced by any outstanding object in general. Therefore, the vortex field at the tail part of the truncated model was kept basically consistent with that of the complete model. Therefore, the paper only researched the cabin model. In addition, the vortex field in the tail would not influence the noise in cabin. In addition, by applying the truncated model in the research, the computational time can be saved, and the computational efficiency can be improved. At present, this method has been adopted in some published papers. For example, Xiao [13] used a truncated model to research the aerodynamic noise in a high-speed train cabin. However, the research results in reference [13] were not refined and verified by experiments.

The high-quality element was critical for improving the computational precision regarding transient flow field of the head surface and improving the convergence process. The structural element based on the mapping method was featured with high computational efficiency and ordered arrangement, thus being an ideal meshing method. According to quadrilateral mesh method of the head generated by the mapping method, the three-dimensional modeling software CATIA was adopted to output the appearance of complex high-speed train as the mapping reference, and the quadrilateral element was mapped to the streamlined shape surface of the high-speed train, which could save the simplification process for the streamlined shape and could divide elements very orderly. Based on the generated quadrilateral element of the high-speed train surface, a hexahedral mesh used for flow field could be formed easily. The geometric model can be found in Fig. 3. The fluctuating pressure on the train surface was relatively small when the analyzed frequency was more than $1000 \mathrm{~Hz}$ [10]. Therefore, the maximum computation frequency was set as $1000 \mathrm{~Hz}$ when the flow field around the train head was simulated numerically by LES method. In the computation, the fluctuating pressure of each node on the body surface was monitored. Strong pressure gradient was in the nose tip, in the lower front window of the cabin, and in the transition position from the head surface to the roof. Therefore, in the lines of the longitudinal symmetrical plane of the head surface, three observation points were selected in the nose tip, in the lower front window of the cabin, and in the transition position from the head surface to the roof as key objects for discussions.

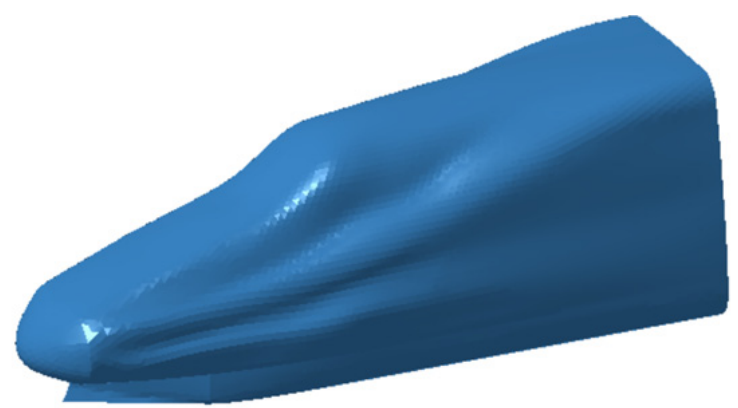

Fig. 3. Geometric model of the high-speed train cabin

Based on the finite volume method, the commercial software Fluent was applied for analysis and adopted to make numerical simulation of unstructured elements. The turbulent flow was solved based on LES in Fluent framework and through the application of Smagorinsky-Lilly subgrid model. The non-viscidity condition was applied in two lateral sides, sliding boundary condition was applied in the roof surface, and fixed boundary condition was applied in the floor. The inlet of the computational domain was designated as the velocity inlet, whose speed was set as $250 \mathrm{~km} / \mathrm{h}$. Such speed was corresponding to the Reynolds number $R_{e}=1.23 \times 10^{7}$. The outlet 
was designated as the pressures outlet, as shown in Fig. 4. The outer surface of the cabin was extracted to obtain the flow elements using HYPERMESH. Flow elements on the outer surface were triangular element, and there were 50672 elements and 57825 nodes. The material was steel, and elastic modulus was $2.1 \mathrm{e}^{11}$, density was $7.8 \mathrm{e}^{3} \mathrm{~kg} / \mathrm{m}^{3}$, and Poisson's ratio was 0.31 .

RANS framework could be applied for the computation, in order to realize the $k-\varepsilon$ eddy viscosity transmission model and obtain the steady solution of the flow field. Based on such steady solution, LES was applied for the unsteady computation of the flow field. In consideration of two factors of the computational accuracy and efficiency, the unsteady time step was determined as $5 \times 10^{4} \mathrm{~s}$, and twenty-five sub-iterations were conducted each time. Through the analysis of residuals, the pressure coefficients of rear mirror and body surface, rear mirror speed and other parameters can be monitored, the computational convergence in each time step was determined, and sufficient sample points in the quasi-periodic flow could be ensured by the set time step. The process of change regarding observation points at $0-1 \mathrm{~s}$ was analyzed, finding that dynamic steady flow was determined in the computational time of more than $1 \mathrm{~s}$. A total of 4000 time steps were required for the entire computation, and the results of the final 2000 time steps were applied for the unsteady data analysis. It should be noted that the time step required for the computation can ensure the enough points of all observation points in the quasi-periodic period. Meanwhile, the analysis of the final $1 \mathrm{~s}$ data can guarantee that standard values of average pressure factor and fluctuating pressure factor brought from the computation had the maximum error of $0.5 \%$.

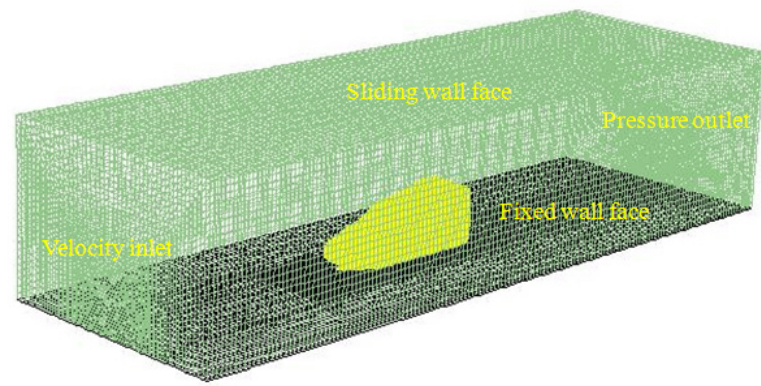

Fig. 4. Computational domain of the high-speed train cabin

The fluctuating pressure $P_{v}$ on the train surface was related to the pulsating pressure level. The fluctuating pressure level $L_{v}$ was defined as follows:

$L_{v}=10 \lg \left(P_{v} / P_{0}\right)^{2}$,

wherein: $P_{0}$ was the reference pressure with the value of $2 \times 10^{5} \mathrm{~Pa}$.

The fluctuating pressure level obtained through Eq. (15) was shown in Fig. 5. It was indicated that the fluctuating pressure level at observation point 1 and point 2 was significantly greater than that at observation point 3 . And obvious peaks and valleys were presented in the fluctuating pressure level at various observation points. The pressure contours of the high-speed train cabin at $0.125 \mathrm{~s}, 0.250 \mathrm{~s}, 0.375 \mathrm{~s}$ and $0.500 \mathrm{~s}$ were extracted as shown in Fig. 6. As presented in Fig. 9, the relatively large pressure was mainly appeared in the transition position and at the bogie. Fig. 7 was the velocity contour of the high speed train cabin. With the increase of the analyzed time, the velocity in the tail of the high speed train has an obvious change.

\section{Computation of aerodynamic noises of the high-speed train}

Acoustic analysis software Virtual.lab was applied in the paper to establish an acoustic boundary element model of the high-speed train cabin. Due to the unavailable modeling capabilities in the pretreatment of Virtual.lab software, the finite element model of sound field of 
the cabin cavity was firstly established in HYPERMESH and then imported to the Virtual.lab in BDF format. Before modeling, the size of acoustic elements should be determined; whose ideal size was approximately six elements in each wavelength. The boundary element model of the high-speed train cabin was finally obtained as shown in Fig. 8.

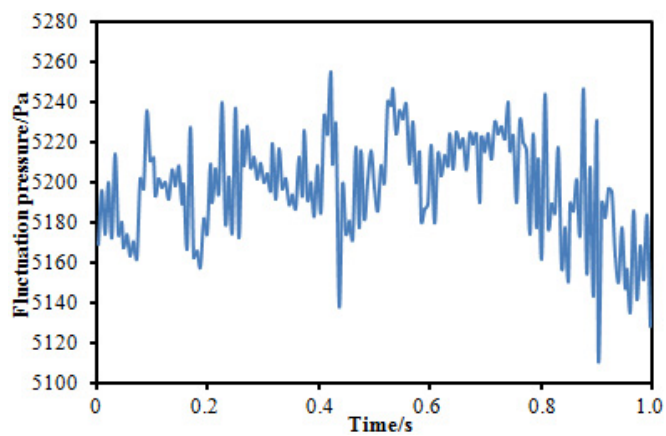

a) Fluctuating pressure level at point 1

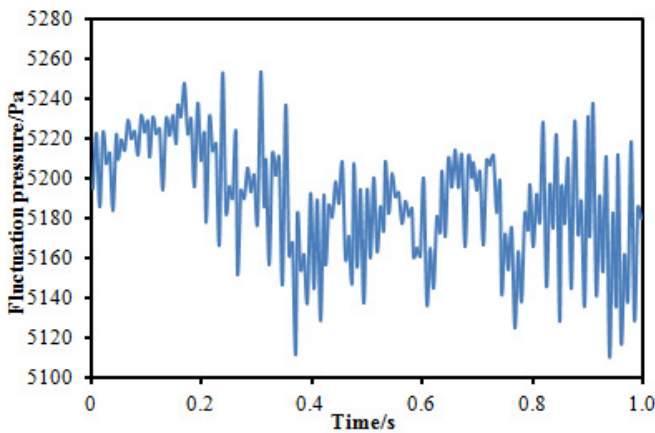

b) Fluctuating pressure level at point 2

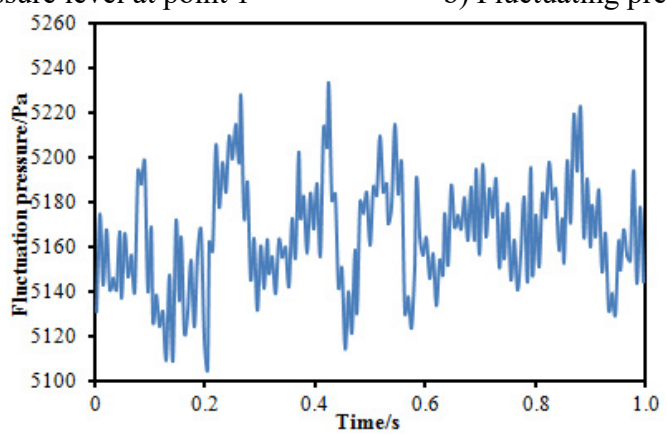

c) Fluctuating pressure level at point 3

Fig. 5. Fluctuating pressure level at three observation points

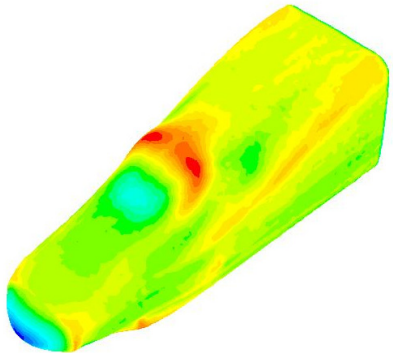

a) $0.125 \mathrm{~s}$

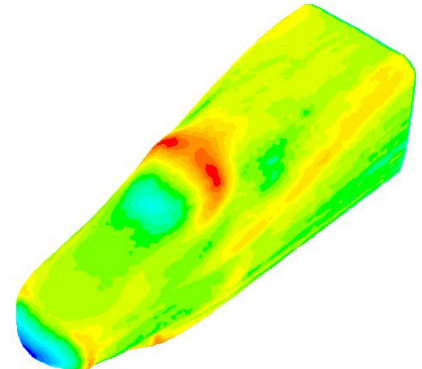

c) $0.375 \mathrm{~s}$

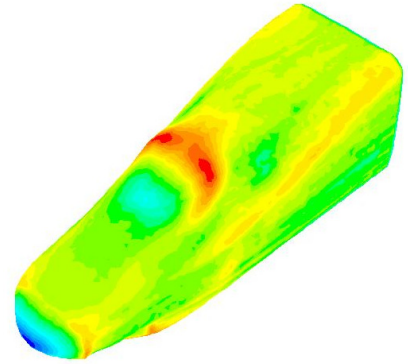

b) $0.250 \mathrm{~s}$

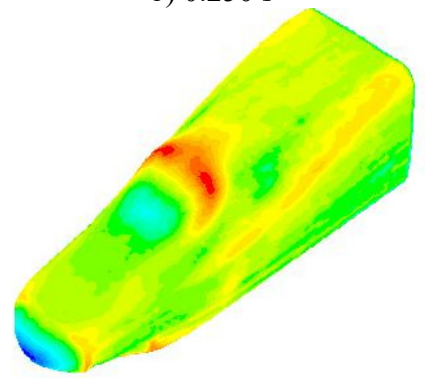

d) $0.500 \mathrm{~s}$

Fig. 6. Fluctuating pressure contours of the high-speed train cabin 


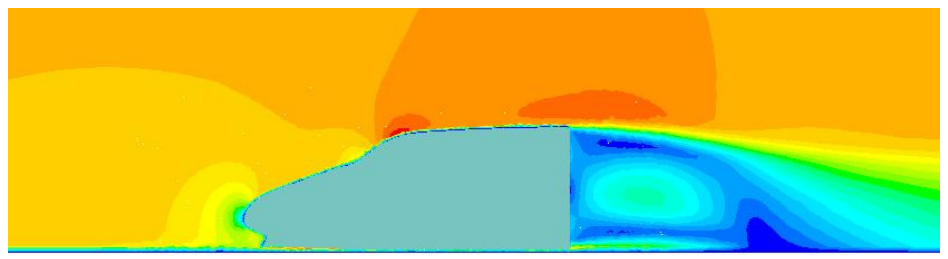

a) $0.125 \mathrm{~s}$

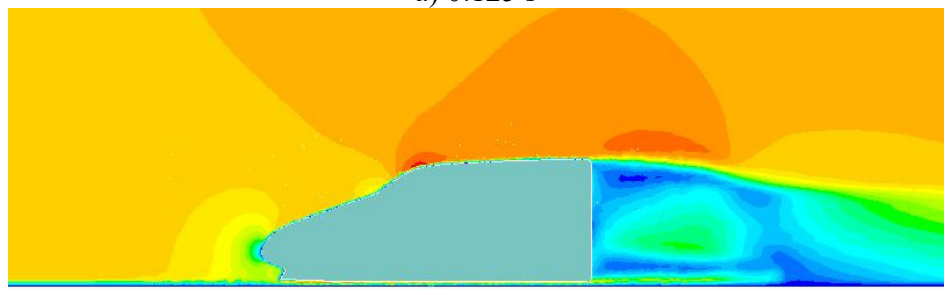

b) $0.250 \mathrm{~s}$

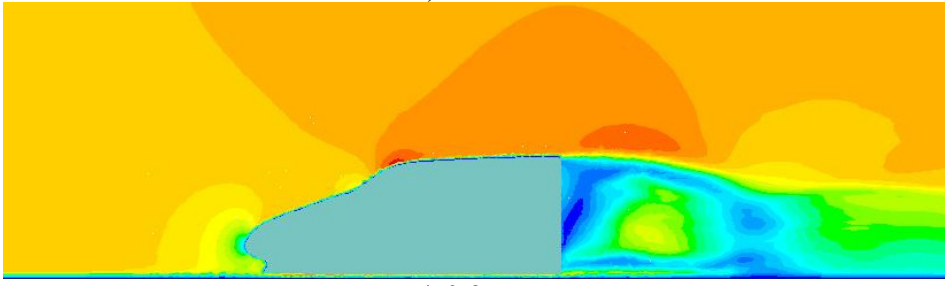

c) $0.375 \mathrm{~s}$

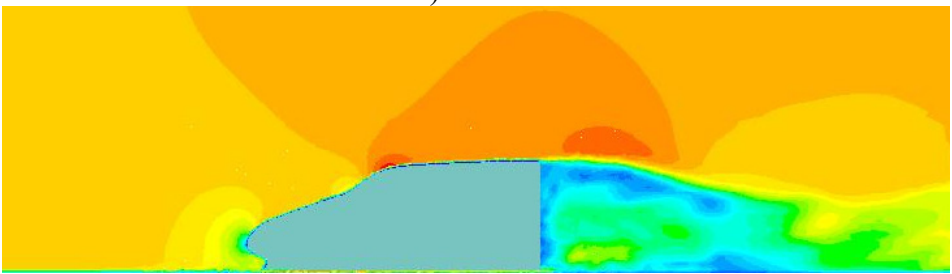

d) $0.500 \mathrm{~s}$

Fig. 7. Velocity contours of the high-speed train cabin

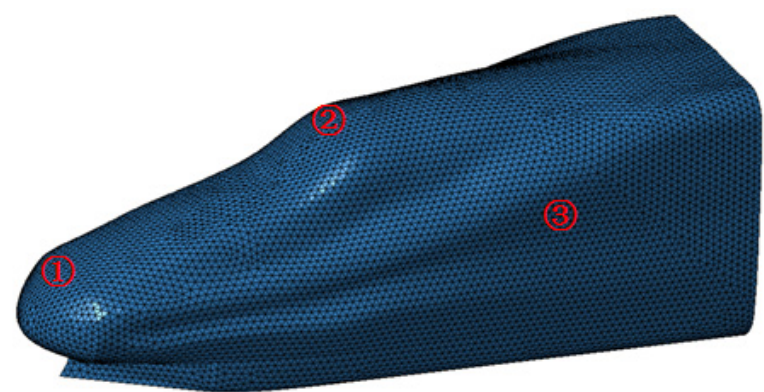

Fig. 8. Boundary element model of the high-speed train cabin

The aerodynamic characteristics in Section 3 were imported into Virtual.lab and the computational result was mapped to the boundary element meshes. Therefore, all characteristics of structural meshes could be obtained through acoustic elements, thereby obtaining acoustic results. Similarly, three observation points were arranged at the cabin nose tip, in the transition position from the nose tip to the roof and in the windows, and their SPLs were extracted, as shown in Fig. 9. It could be seen that SPLs at the observation points 1 and 2 were significantly larger than that of observation point 3. In addition, SPLs at all observation points were gradually decreased with the increase of the analyzed frequency. There were no any obvious peaks in the 
whole observation point. The minimum of the observation point 1 was $62.2 \mathrm{~dB}$ and the corresponding frequency was $587 \mathrm{~Hz}$. The minimum of the observation point 2 was $63.3 \mathrm{~dB}$ and the corresponding frequency was $947 \mathrm{~Hz}$. The minimum of the observation point 3 was $57.1 \mathrm{~dB}$ and the corresponding frequency was $923 \mathrm{~Hz}$.

The SPL contours at $100 \mathrm{~Hz}, 300 \mathrm{~Hz}, 500 \mathrm{~Hz}$ and $700 \mathrm{~Hz}$ were extracted, whose results were shown in Fig. 10. It was indicated that greater SPLs were presented in transitions and bogies, which was consistent with the above result of aerodynamic characteristics.

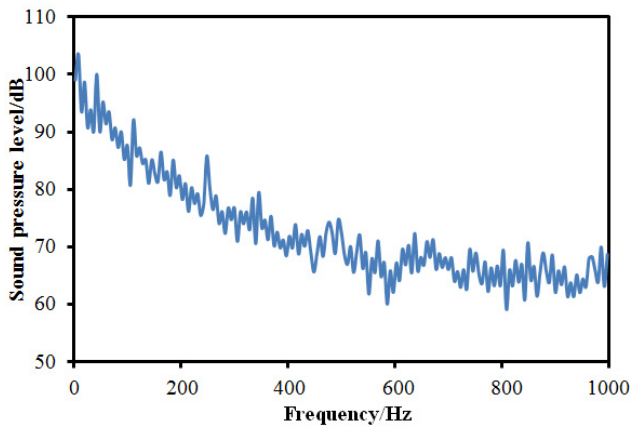

a) SPLs at observation point 1

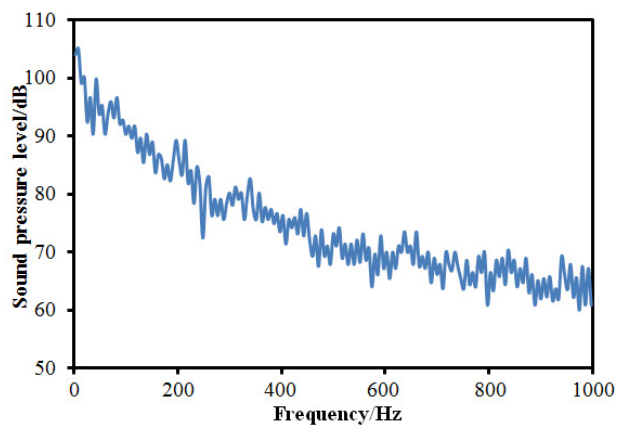

b) SPLs at observation point 2

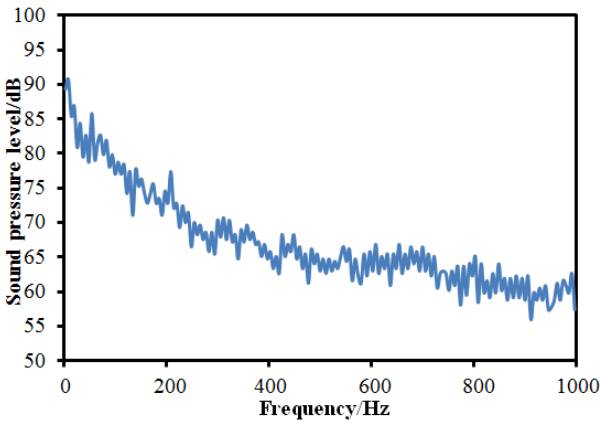

c) SPLs at observation point 3

Fig. 9. SPLs curves at three observation points

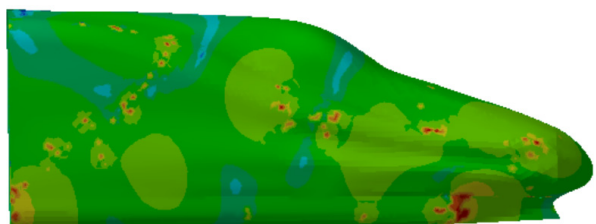

a) $100 \mathrm{~Hz}$

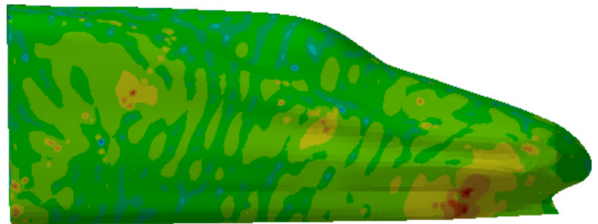

c) $500 \mathrm{~Hz}$

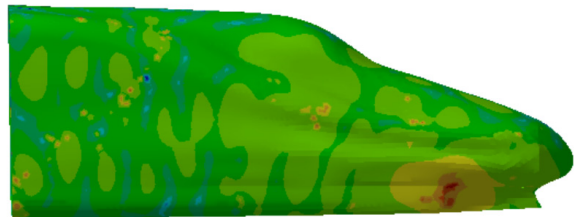

b) $300 \mathrm{~Hz}$

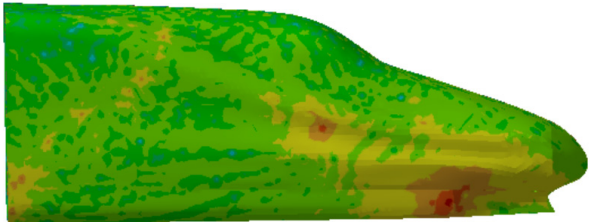

d) $700 \mathrm{~Hz}$

Fig. 10. SPL contours of the high-speed train cabin

\section{Experimental verification of aerodynamic noises of the high-speed train}

The computational model of the aerodynamic noise of the high speed train was relative complex, and it should be verified by the corresponding experiment. The experiment was 
conducted in a wind tunnel. The cross-sectional area was $8 \mathrm{~m} \times 6 \mathrm{~m}$ and the length was $15 \mathrm{~m}$. The wind speed was controlled in $20 \mathrm{~m} / \mathrm{s}-70 \mathrm{~m} / \mathrm{s}$. To reduce the influence of the floor boundary layer, the special floor for the train was mounted in the test section. A turntable with the diameter of $7 \mathrm{~m}$ and rotating range of $360^{\circ}$ was placed in the middle of the floor, while other parts were fixed. The upper surface of the floor was $1.06 \mathrm{~m}$ away from the lower wind tunnel wall, and the center of the turntable was $7.84 \mathrm{~m}$ away from the front edge of the floor and $8.26 \mathrm{~m}$ away from the rear edge. The front and rear edges of the floor were processed to the streamlined shape to reduce the disturbance of airflow and inclined gaps between floors. Due to limitations of sizes of the wind tunnel, the scaling high-speed train was applied in the experimental process.

Three microphones were arranged in the head train to measure the sound pressure level of the observation point, as shown in Fig. 11. The measurement flow chart diagram was presented in Fig. 12. Bruel \& Kjaer measurement instrumentation was used to collect the signal and then it was connected to the computer. The sampling frequency was $8000 \mathrm{~Hz}$. The collected signal was processed by the Pulse software to obtain the sound pressure level. Each experiment was conducted three times to obtain the average value as the final result. Finally, the experimental result was compared with the simulation as shown in Fig. 13. It was indicated that the experimental SPLs were generally larger than the numerical simulation results mainly because the inflow in simulation process was set to be uniform flow, which could not be achieved in the experimental process. Besides, some details of the geometric model were cleared in the simulation process, thus resulting in some differences with the actual model. Finally, these factors would cause some errors which were within $5 \mathrm{~dB}$, and errors can be accepted by the actual engineering. The prediction model can be considered effective.

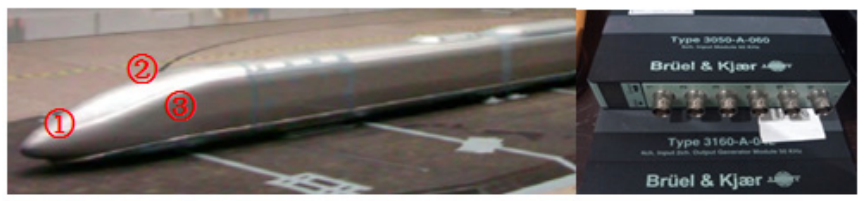

Fig. 11. Observation points and equipment

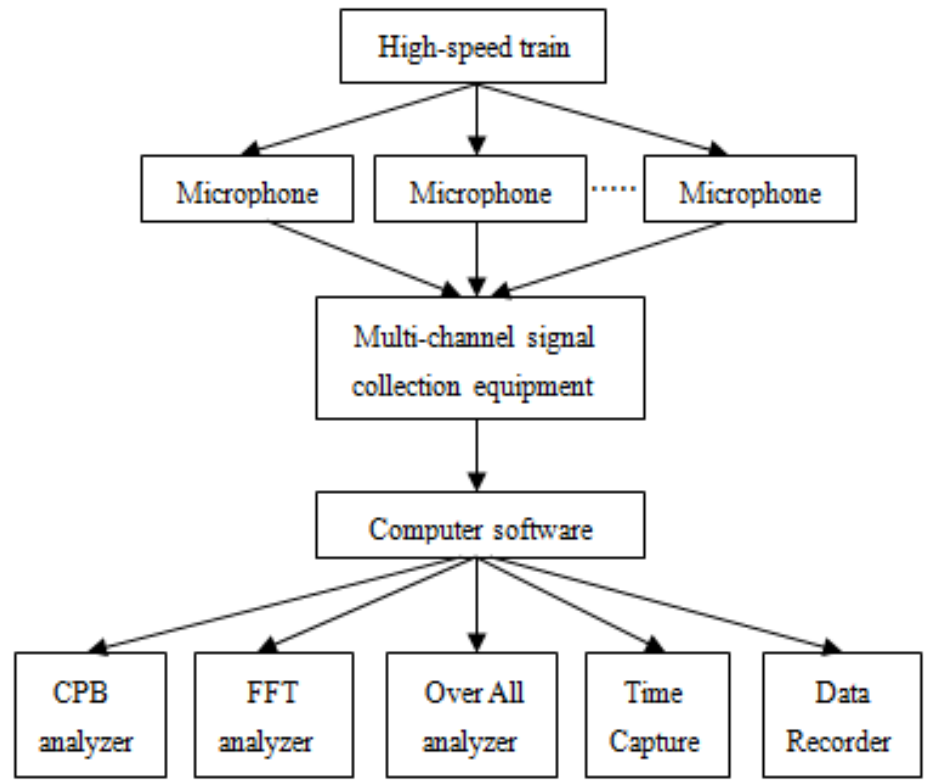

Fig. 12. Experimental process of aerodynamic noises of the cabin 


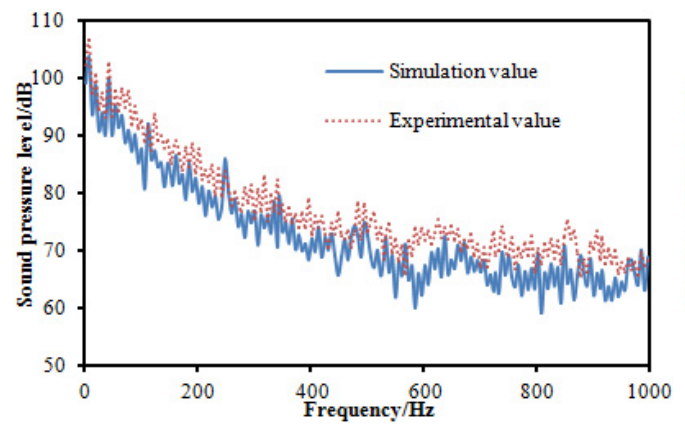

a) SPL comparison between experiment and simulation at observation point 1

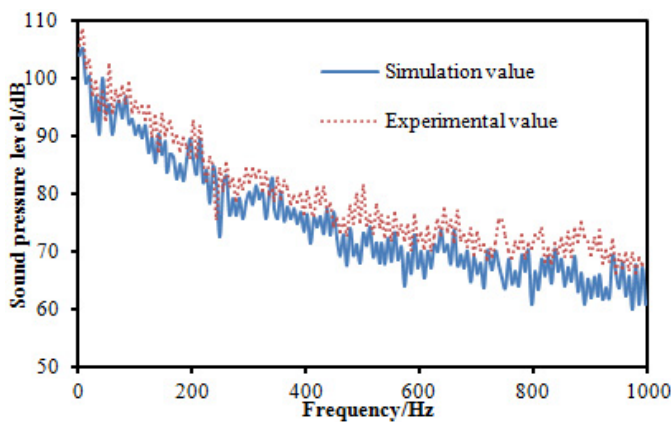

b) SPL comparison between experiment and simulation at observation point 2

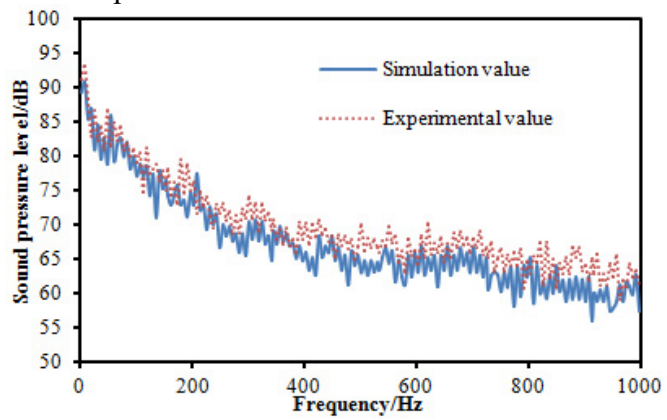

c) SPL comparison between experiment and simulation at observation point 3

Fig. 13. SPL comparison between experiment and simulation at three observation points

\section{Conclusions}

For improving noises in the cabin quickly, large eddy simulation was adopted to compute the fluctuating pressure of the cabin surface numerically. Then, the fluctuating pressure was mapped to the boundary element model as the excitation loads to obtain the aerodynamic noise distribution of the cabin at different frequencies, which was then compared with the experimental result. There were some differences between the computational model and actual model, and the experimental results were more than the simulation result, which was within the acceptable range of the engineering. The comparative result indicated that the prediction model was reliable. In addition, as presented from the computational results: SPLs on cabin surface were changed between $60 \mathrm{~dB}-110 \mathrm{~dB}$, and greater SPLs were located on the transition position which was from the nose tip to the roof surface of the cabin. The aerodynamic noise on the cabin surface was mainly in low-frequency.

\section{References}

[1] Shen Z. Y. Dynamic environment of high-speed train and its distinguished technology. Journal of the China Railway Society, Vol. 28, Issue 4, 2006, p. 1-5.

[2] Goldstein M. V. Unified approach to aerodynamic sound generation in the presence of boundaries. Journal of the Acoustical Society of America, Vol. 56, Issue 2, 1974, p. 497-509.

[3] Kitagawa T., Nagakura K. Aerodynamic noise generated by Shin Kansen cars. Journal of Sound and Vibration, Vol. 231, Issue 5, 2000, p. 913-924.

[4] Sassa T., Sato T., Yatsui S. Numerical analysis of aerodynamic noise radiation from a high-speed train surface. Journal of Sound and Vibration, Vol. 247, Issue 3, 2001, p. 407-416.

[5] Nagakura K. Localization of aerodynamic noise sources of Shin Kansan trains. Journal of Sound and Vibration, Vol. 293, Issue 3, 2006, p. 547-556.

[6] Liu H. G., Lu S. L., Zeng F. L. Experimental study of automobile aerodynamic noise. China Journal of Highway and Transport, Vol. 18, Issue 1, 2005, p. 113-116. 
[7] Liu H. G., Lu S. L. Calculation method of the aerodynamic noise around high speed vehicles. Journal of Traffic and Transportation Engineering, Vol. 2, Issue 2, 2002, p. 41-44.

[8] Liu J. L., Zhang J. Y., Zhang W. H. Numerical analysis on aerodynamic noise of the high-speed train head. Journal of the China Railway Society, Vol. 33, Issue 9, 2011, p. 19-26.

[9] Xiao Y. G., Kang Z. C. Numerical prediction of aerodynamic noise radiated from high speed train head surface. Journal of Central South University, Vol. 39, Issue 6, 2008, p. 1267-1272.

[10] Yuan L., Li R. X. Aerodynamic noise of high-speed train and its impact. Mechanical Engineering and Automation, Vol. 180, Issue 5, 2013, p. 31-35.

[11] Yang Y., Yang G. W. A numerical study on aerodynamic noise sources of high-speed train. Proceedings of the 1st IWHIR, Vol. 2, 2012, p. 107-116.

[12] Sun Z. X., Song J. J., An Y. R. Numerical simulation of aerodynamic noise generated by CRH3 high speed trains. Acta Scientiarum Naturalium Universitatis Pekinensis, Vol. 48, Issue 5, 2012, p. 701-711.

[13] Xiao Y. G., Tian H. Q., Zhang H. Prediction of interior aerodynamic noise of high-speed train cab. Journal of Traffic and Transportation Engineering, Vol. 8, Issue 3, 2008, p. 10-14.

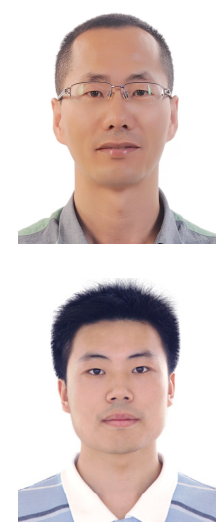

Ming Lv received Master's degree in Ecological engineering from Fujian Agriculture and Forestry University, Fuzhou, China, in 2006. Now he works at Bengbu University. His current research interests include control, dynamics, mechanical design method and theory.

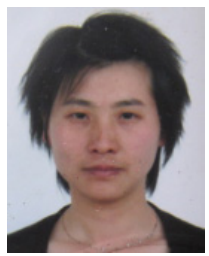

Dong-er Xu received Master's degree in Faculty of Science and Engineering from University of Liverpool, Liverpool City, UK, in 2015. Now he works at Bengbu University. His current research interests include control and additive manufacturing.

Hua He received Doctor's degree in vehicle operation engineering from Central South University, Changsha, China, in 2008. Now she works at Bengbu University. Her current research interests include strength design and finite-element analysis. 